Supporting information for:

Mechanism for Broadband White-Light Emission from Two-Dimensional (110)

\title{
Hybrid Perovskites
}

Te Hu, ${ }^{1,2}$ Matthew D. Smith, ${ }^{3}$ Emma R. Dohner, ${ }^{3}$ Meng-Ju Sher, ${ }^{2}$ M. Tuan Trinh, ${ }^{4}$ Alan Fisher, ${ }^{5}$ Jeff Corbett, ${ }^{5}$ X.-Y. Zhu, ${ }^{4}$ Hemamala I. Karunadasa ${ }^{*}, 3$ and Aaron M. Lindenberg ${ }^{*}, 1,2,6$

${ }^{1}$ Stanford Institute for Materials and Energy Sciences, SLAC National Accelerator Laboratory, Menlo Park, CA 94025, USA

${ }^{2}$ Department of Materials Science and Engineering, Stanford University, Stanford, California 94305, USA

${ }^{3}$ Department of Chemistry, Stanford University, Stanford, California 94305, USA

${ }^{4}$ Department of Chemistry, Columbia University, New York, NY 10027

${ }^{5}$ SLAC National Accelerator Laboratory, Menlo Park, CA 94025, USA

${ }^{6}$ PULSE Institute, SLAC National Accelerator Laboratory, Menlo Park, CA 94025, USA

*aaronl@stanford.edu, hemamala@stanford.edu

Experimental details

Figures S1-S13

References 


\section{Experimental details}

\section{$(N$-MEDA $)\left[\mathrm{PbBr}_{4}\right]$ sample preparation}

Thin films of ( $N$-MEDA)[PbBr 4$]$ were obtained by spin-coating a $0.1 \mathrm{M}$ solution of $(N$ MEDA) $\left[\mathrm{PbBr}_{4}\right]$ in $\mathrm{N}, \mathrm{N}$-dimethylformamide at $3000 \mathrm{rpm}$ on either glass or crystalline quartz substrates, and then annealing at $100{ }^{\circ} \mathrm{C}$ for 10 minutes. Single crystals were grown as previously described, ${ }^{[\mathrm{S} 1]}$ with a typical size of $\sim 400 \times 400 \times 50 \mu \mathrm{m}^{3}$. Pellet samples were obtained by diepressing ball-milled powders with a particle size of $\sim 1 \mu \mathrm{m}$ at a pressure of 10,000-15,000 psi.

\section{Grazing-incidence X-ray Diffraction (GIXD)}

GIXD was carried out at the Stanford Synchrotron Radiation Lightsource (SSRL) at beamline 11-3 with x-ray energy of $12.735 \mathrm{keV}$ and an area detector. X-ray incidence angle is $0.1^{\circ}$. Thin film samples used for the GIXD measurement were on crystalline quartz substrates to minimize the diffuse scattering background. Raw images were processed using WxDiff to obtain the inplane $\left(Q_{x y}\right)$ and out-of-plane $\left(Q_{z}\right)$ diffraction pattern, ${ }^{[\mathrm{S} 2]}$ as shown in Figure S1a.

\section{THz transient photoconductivity measurement}

The measurement was carried out on $(N-\mathrm{MEDA})\left[\mathrm{PbBr}_{4}\right]$ thin films deposited on crystalline quartz substrates, using a home-built setup. In brief, laser pulses (800-nm center wavelength, 1.7-mJ pulse energy, 50-fs pulse duration and $1-\mathrm{kHz}$ repetition rate) from a Ti:sapphire femtosecond regenerative amplifier was split into two paths. The first path was directed either into a frequency-tripling setup to generate 266-nm light or into an optical parametric amplifier to generate 387-nm light as the pump. The second path was used for $\mathrm{THz}$ radiation generation, where $\mathrm{THz}$ probe pulses were generated from a two-color laser-induced gas plasma. Pump light 
traveled collinearly with the $\mathrm{THz}$ probe and overlapped at the surface of the sample, confirmed with a dummy scan on silicon. The transmitted $\mathrm{THz}$ beam was detected by electro-optic sampling in a 1-mm thick ZnTe crystal. A boxcar (SR250, Stanford Research Systems) and lockin amplifier (SR830, Stanford Research Systems) were used to detect the small signal changes of the transmitted THz beam due to photoexcitation.

\section{Transient absorption (TA) measurement}

Transient absorption measurements were carried out on $(N-\mathrm{MEDA})\left[\mathrm{PbBr}_{4}\right]$ thin films on glass substrates. A similar setup was used as for $\mathrm{THz}$ measurement, except that the second path was focused onto a sapphire crystal to produce a white-light supercontinuum probe. Different probe colors were selected using fluorescence band-pass filters. The 387-nm pump traveled collinearly with the probes and overlapped on the sample surface. The pump was lightly focused to a spot of $1.2 \times 1.2 \mathrm{~mm}^{2}$ on the sample surface to ensure probe light was within the pump. The transmitted probe beam was collected using a fast Si photodiode (DET100A, Thorlabs, Inc.). A boxcar (SR250, Stanford Research Systems) and lock-in amplifier (SR830, Stanford Research Systems) were used to detect the small signal change of the transmitted probe beam. The time resolution is $\sim 90 \mathrm{fs}$, as measured by cross-correlation of the white-light supercontinuum with $800-\mathrm{nm}$ laser light.

\section{Streak camera measurement}

A fiber laser (output 1030-nm wavelength, 500-fs pulse duration and 1.28-MHz repetition rate) was synchronized with a streak camera (C5680, Hamamatsu) through a common 119-MHz source (CG635, Stanford Research Systems). The laser output was frequency-tripled to $343 \mathrm{~nm}$ 
as pump and the streak camera was operated in "Synchroscan" mode. PL was either sent through band-pass filters to select individual emission wavelengths or alternatively dispersed using a grating onto the streak camera slit to measure all wavelengths at once.

\section{Kerr-gate ultrafast PL measurement}

Ultrafast PL spectroscopy was performed using a home-built Kerr-gate setup. ${ }^{[33]}$ In brief, 800$\mathrm{nm}$ pulses from a Ti:sapphire femtosecond regenerative amplifier was split into two paths. One path was used for sample excitation after frequency-tripling to $266 \mathrm{~nm}$ and the other path was used to rotate the polarization of the PL at a Kerr medium (1-mm thick SFL-6 glass, SCHOTT AG), which was placed between two crossed film polarizers. Off-axis parabolic mirrors were used for luminescence collection. Luminescence from the ( $N$-MEDA) $\left[\mathrm{PbBr}_{4}\right]$ pellet after passing through the first polarizer was focused into the Kerr medium together with gating pulses, which came in at an oblique angle and were variably delayed by a translation stage. PL then passed through the second polarizer and was focused into a photomultiplier module (PMM01, Thorlabs, Inc.) with color filters used to select the probed wavelengths. A boxcar (SR250, Stanford Research Systems) and lock-in amplifier (SR830, Stanford Research Systems) were used to detect the small PL signal. The system has a time resolution of $\sim 1.3 \mathrm{ps}$.

\section{Time-Correlated Single Photon Counting (TCSPC) measurement}

Measurement was performed using a TCSPC system (TimeHarp 260 PICO, PicoQuant). A single crystal and pellet sample of $(N-\mathrm{MEDA})\left[\mathrm{PbBr}_{4}\right]$ were excited using a fiber laser (frequencytripled from a fundamental wavelength of $1030 \mathrm{~nm}$ to $343 \mathrm{~nm}, 500-\mathrm{fs}$ pulse duration and 1.28-MHz repetition rate), and PL was detected using a hybrid photomultiplier detector assembly 
(PMA Hybrid 06, PicoQuant). Detection wavelengths were selected using fluorescence bandpass filters (Semrock, Inc.) and excitation fluence was controlled using reflective neutral density filters (NDK01, Thorlabs, Inc.). The response function of the system has a full width at halfmaximum (FWHM) of $\sim 120$ ps.

\section{PL spectrum measurement}

PL from ( $N$-MEDA) $\left[\mathrm{PbBr}_{4}\right]$ and $\left(N\right.$-MPDA) $\left[\mathrm{PbBr}_{4}\right]$ samples was collected with a spectrograph (Acton Research SpectraPro 500i) equipped with a silicon CCD (Hamamatsu), using excitation from a $375 \mathrm{~nm} \mathrm{CW}$ diode laser. The excitation intensity was $23.7 \mathrm{~mW} / \mathrm{cm}^{2}$. Additional spectra were collected on a Horiba Jobin-Yvon Spex Fluorolog-3 fluorimeter equipped with a $450 \mathrm{~W}$ xenon lab and a thermoelectrically-cooled R928P detector. Incidence light was passed through a double-grating monochromator and data was collected using the FluorEssence 2.3.15 software.

(a)

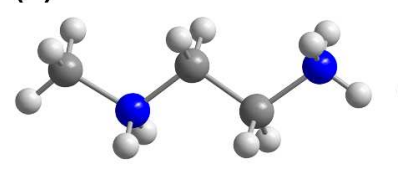

(b)

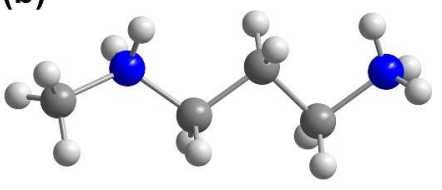

Figure S1. Molecular structures of $N$-MEDA (left) and $N$-MPDA (right) cations in $(N-$ MEDA) $\left[\mathrm{PbBr}_{4}\right]$ and $(N-\mathrm{MPDA})\left[\mathrm{PbBr}_{4}\right]$. The organic cation in $(N-\mathrm{MPDA})\left[\mathrm{PbBr}_{4}\right]$ is disordered across two positions: only the major component is shown for clarity. Blue, grey and white spheres represent $\mathrm{N}, \mathrm{C}$, and $\mathrm{H}$ atoms, respectively. 


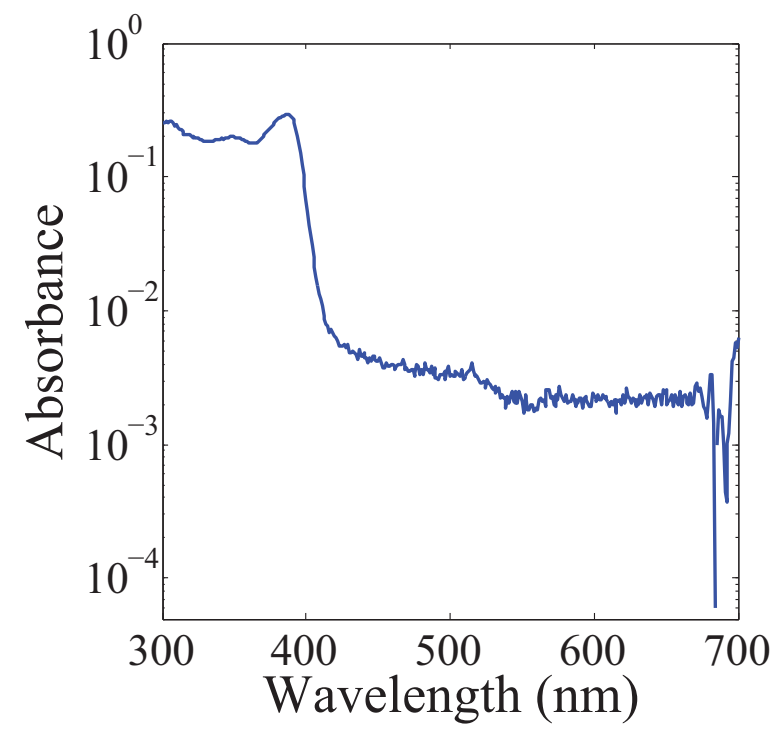

Figure S2. Log plot of the static optical absorption spectrum of a film of ( $N$-MEDA) $\left[\mathrm{PbBr}_{4}\right]$ measured using an integration sphere.

Figure S3a shows $Q_{x y}-Q_{z}$ diffraction pattern of ( $N$-MEDA) $\left[\mathrm{PbBr}_{4}\right]$ thin film on crystalline quartz substrate, obtained from the raw GIXD image using WxDiff. From Bragg's law, we know

$$
\overrightarrow{k^{\prime}}-\vec{k}=\vec{Q}=\overrightarrow{Q_{x y}}+\overrightarrow{Q_{z}}
$$

where $\vec{k}$ and $\overrightarrow{k^{\prime}}$ are the wave vectors of incident and diffracted X-ray beams respectively, and $\vec{Q}$ is the momentum transfer. As Bragg's law is satisfied,

$$
\vec{Q}=2 \pi \vec{G}=2 \pi\left(H \overrightarrow{a^{*}}+K \overrightarrow{b^{*}}+L \overrightarrow{c^{*}}\right),
$$

where $\vec{G}$ is a vector in the reciprocal space, $\mathrm{H}, \mathrm{K}, \mathrm{L}$ are the Miller indices and $\overrightarrow{a^{*}}, \overrightarrow{b^{*}}$ and $\overrightarrow{c^{*}}$ are three reciprocal lattice vectors. Then

$$
\overrightarrow{Q_{x y}}+\overrightarrow{Q_{z}}=2 \pi\left(H \overrightarrow{a^{*}}+K \overrightarrow{b^{*}}+L \overrightarrow{c^{*}}\right) .
$$

From the $Q_{x y}-Q_{z}$ pattern, it can be determined that $\left|\Delta \vec{Q}_{z}\right|=2 \pi / d_{001}=2 \pi\left|\overrightarrow{c^{*}}\right|$ between two adjacent diffraction dots in the same vertical line. This indicates that 


$$
\begin{gathered}
\overrightarrow{Q_{x y}}=2 \pi\left(H \overrightarrow{a^{*}}+K \overrightarrow{b^{*}}\right), \\
\overrightarrow{Q_{z}}=2 \pi L \overrightarrow{c^{*}} .
\end{gathered}
$$

As $\left(N\right.$-MEDA) $\left[\mathrm{PbBr}_{4}\right]$ adopts an orthorhombic structure, this implies that in the spin-coated thin film, the out-of-plane grain stacking is along [001] direction, while there is no preferred in-plane grain orientation. Figure S3b shows the X-ray diffraction pattern of the same sample taken in symmetric geometry, where only lattice planes parallel to the sample surface might satisfy Bragg's law. Only $(00 L)$ planes show up in the diffraction pattern, which confirms the out-ofplane stacking is along [001] in the thin film of this (110) perovskite, as previously seen in (001) two-dimensional perovskite films. ${ }^{[\mathrm{S} 4]}$
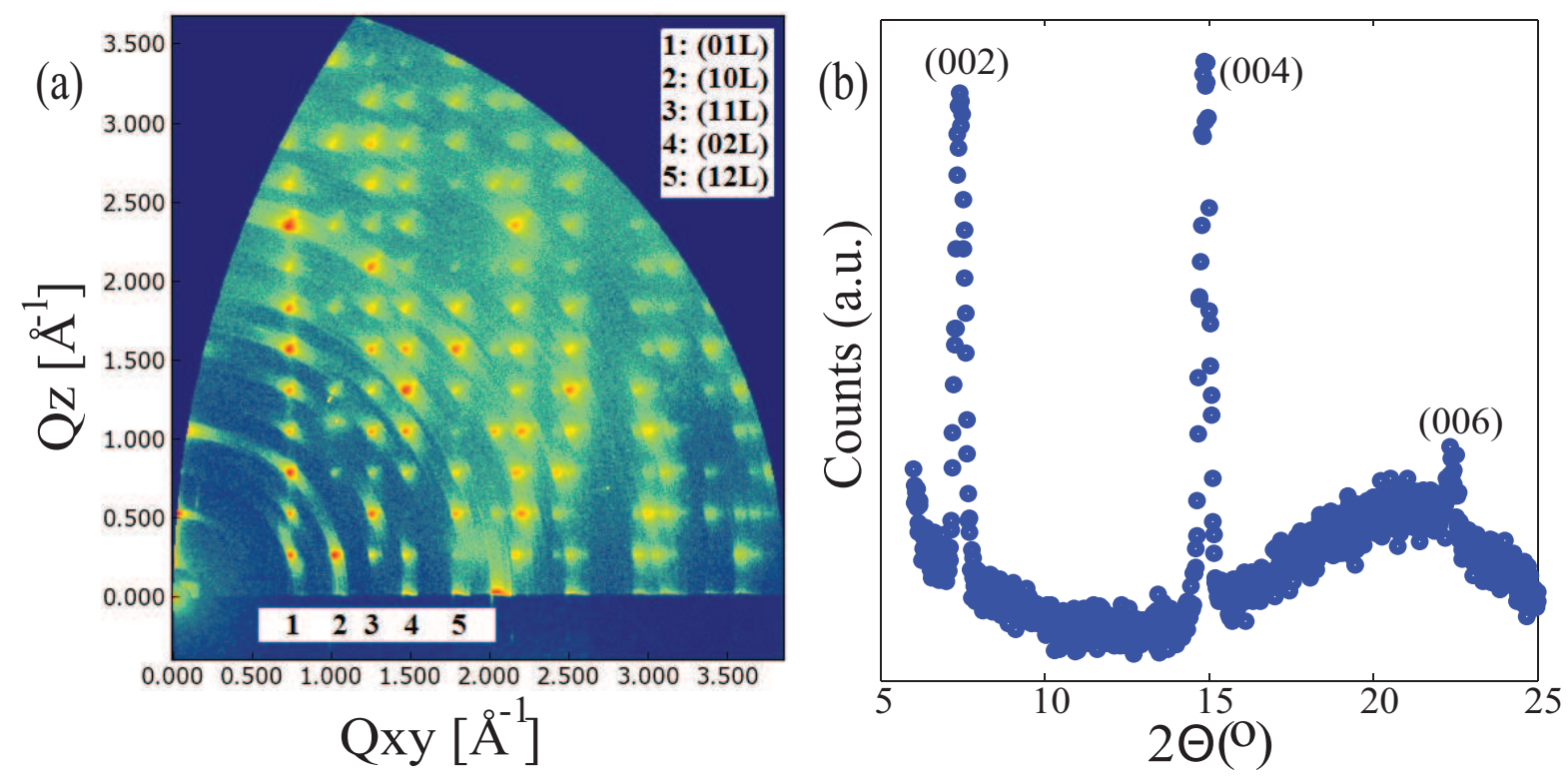

Figure S3. (a) $Q_{x y}-Q_{z}$ diffraction pattern of a (N-MEDA) $\left[\mathrm{PbBr}_{4}\right]$ thin film on crystalline quartz substrate at room temperature. The Miller indices of the first five "lines of dots" are labeled in the upper right corner. (b) X-ray diffraction pattern of the same sample taken in symmetric geometry. Miller indices of the diffraction peaks are labeled on the figure. 


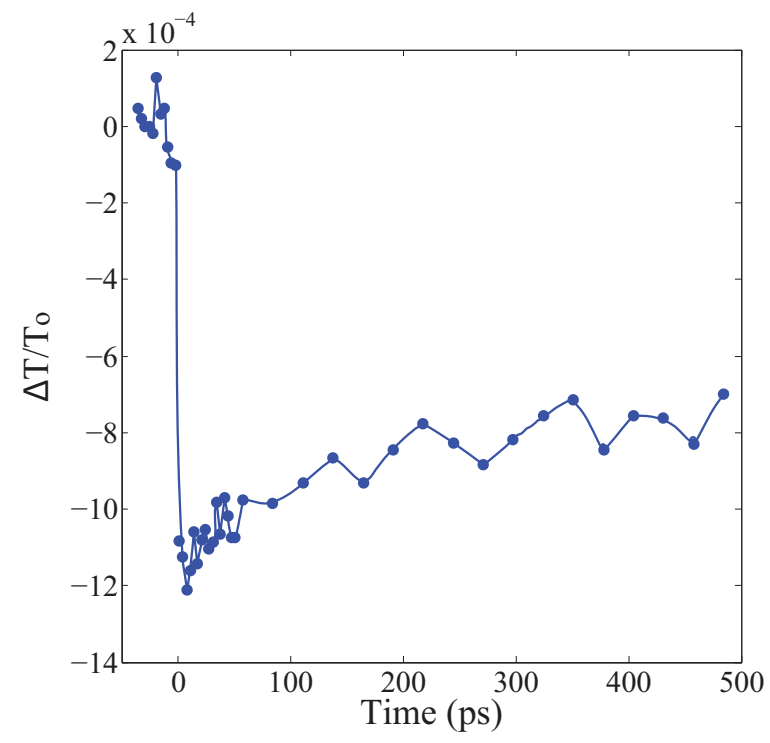

Figure S4. A long time range TA profile with $387-\mathrm{nm}$ photoexcitation and 800-nm optical probe. The excitation fluence is $50 \mu \mathrm{J} / \mathrm{cm}^{2}\left(9.7 \times 10^{13}\right.$ photons $\left./ \mathrm{cm}^{2} / \mathrm{pulse}\right)$.

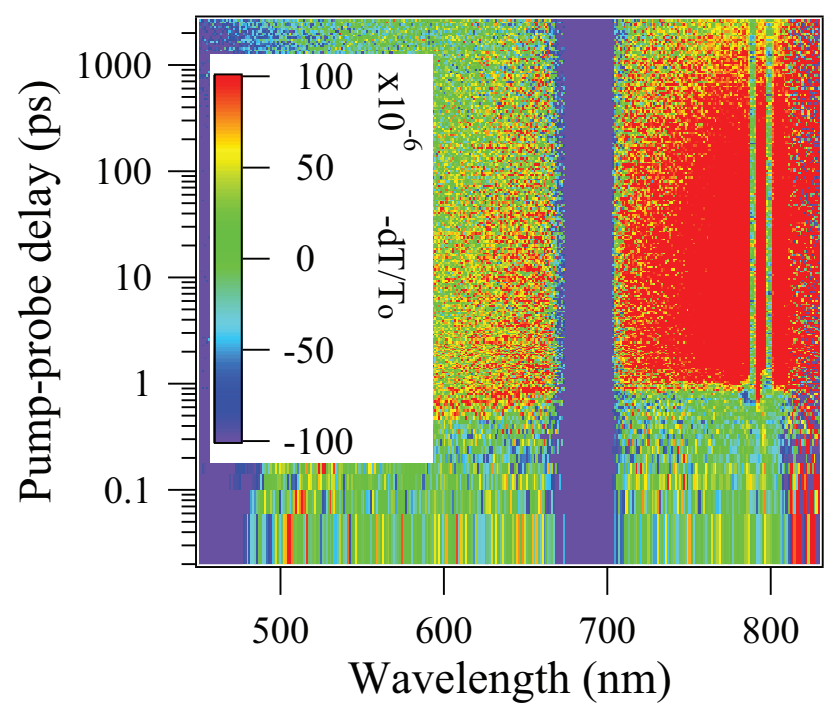

Figure S5. Two-dimensional pseudocolor $\left(-\Delta T / T_{\mathrm{o}}\right)$ plot of the TA spectrum as a function of probe wavelength and pump-probe delay for $(N-\mathrm{MEDA})\left[\mathrm{PbBr}_{4}\right]$. The blank feature around 700 $\mathrm{nm}$ is due to blocking of residual laser light, which was used for $2^{\text {nd }}$ harmonic generation. The excitation wavelength is $345 \mathrm{~nm}$ and the fluence is $\sim 50 \mu \mathrm{J} / \mathrm{cm}^{2}\left(8.7 \times 10^{13}\right.$ photons $/ \mathrm{cm}^{2} /$ pulse $)$.

Figure S6a shows the static absorption and PL spectra from a (N-MPDA) $\left[\mathrm{PbBr}_{4}\right]$ thin film. The narrow free exciton emission with slight asymmetry is dominant in the PL spectrum. The 
absence of lower-energy absorption suggests that self-trapped states have not formed in this material, which does not exhibit broad PL. The weak tail in the exciton towards lower energy and the bleach just below the exciton energy seen in the TA measurements has been previously attributed to permanent trap states in a (001) lead-iodide perovskite. ${ }^{[S 5]}$
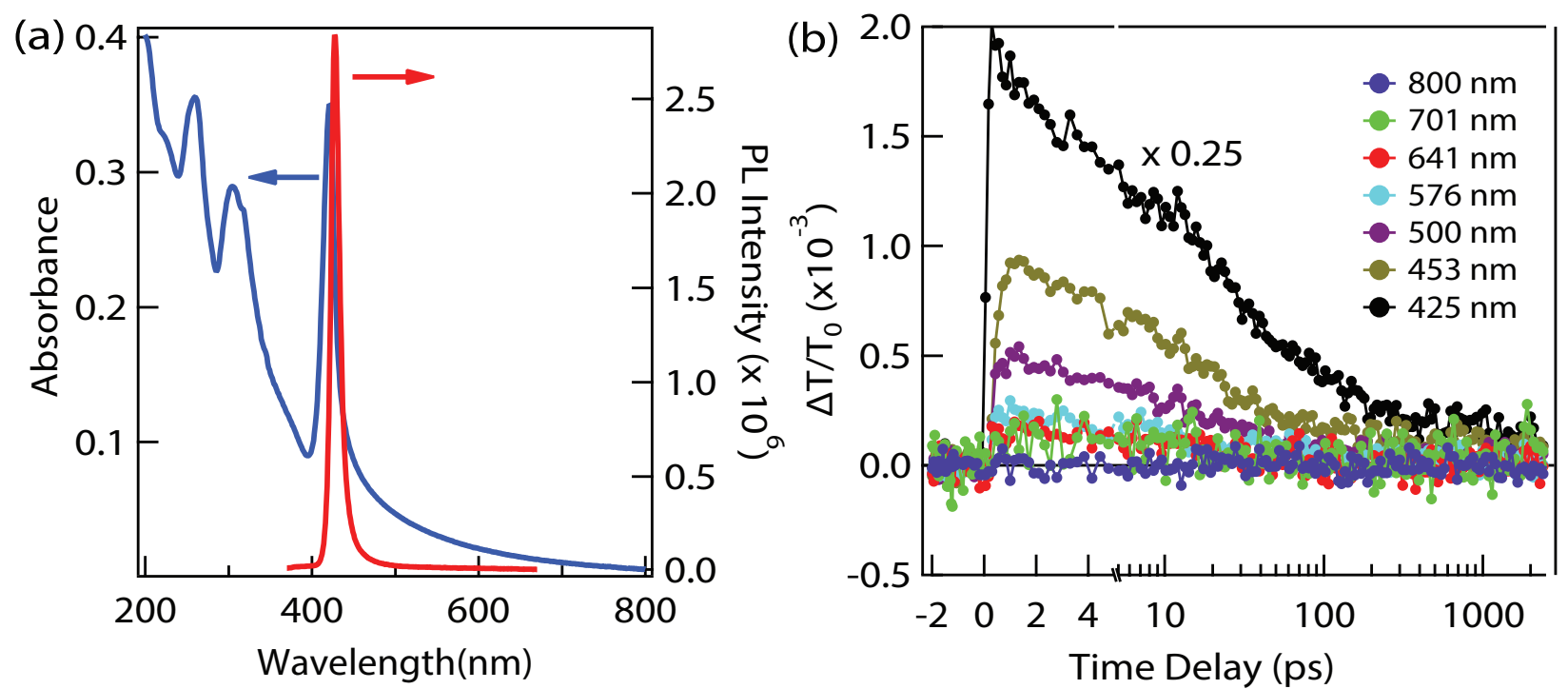

Figure S6. (a) Static absorption and PL spectra from a spin-coated ( $N$-MPDA) $\left[\mathrm{PbBr}_{4}\right]$ thin film on quartz substrate. The PL emission was collected with excitation at $350 \mathrm{~nm}$. (b) TA dynamics probed at different wavelengths on the (N-MPDA) $\left[\mathrm{PbBr}_{4}\right]$ thin film with excitation at $345 \mathrm{~nm}$. Note that the $425-\mathrm{nm}$ trace was multiplied by a factor of 0.25 for better presentation. $\Delta T$ is the transmission difference induced by the pump and $T_{o}$ is the transmission without the pump. The pump fluence in this measurement is $70 \mathrm{uJ} / \mathrm{cm}^{2}\left(1.2 \times 10^{14}\right.$ photons $/ \mathrm{cm}^{2} /$ pulse $)$.

Figure S7 compares the emission spectra and 448-nm PL time traces (measured using TCSPC) of $(N$-MEDA $)\left[\mathrm{PbBr}_{4}\right]$ single crystals and pellet samples at room temperature. Both samples give similar broadband emission. Together with the similar decay dynamics observed in Figure S7b, this indicates that defects introduced during material processing do not significantly contribute to white-light emission. 

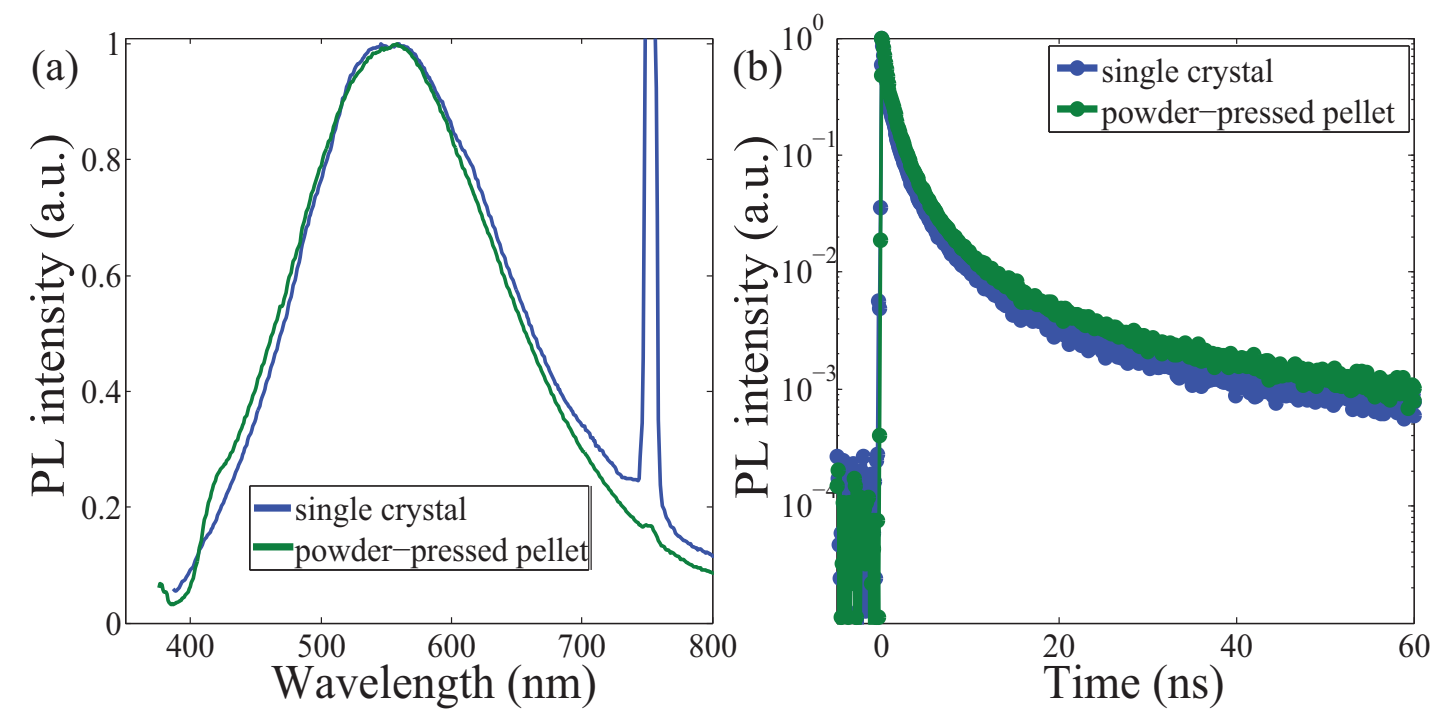

Figure S7. (a) Emission spectra of a $(N-\mathrm{MEDA})\left[\mathrm{PbBr}_{4}\right]$ single crystal and a pellet sample at room temperature. The two peaks at $750 \mathrm{~nm}$ are from residual laser light, which was better blocked in the measurement on the pellet. (b) Time evolution of the 448-nm PL measured using TCSPC at room temperature.

Figure S8 shows the two-axis plot of PL from a pressed pellet sample, as measured by a streak camera. Luminescence from the sample was dispersed by a 1200 -grooves/mm reflective holographic grating (GH25-12V, Thorlabs, Inc.) and projected onto the entrance slit of the streak camera. For longer emission wavelength, the observed PL decays on a longer time-scale.

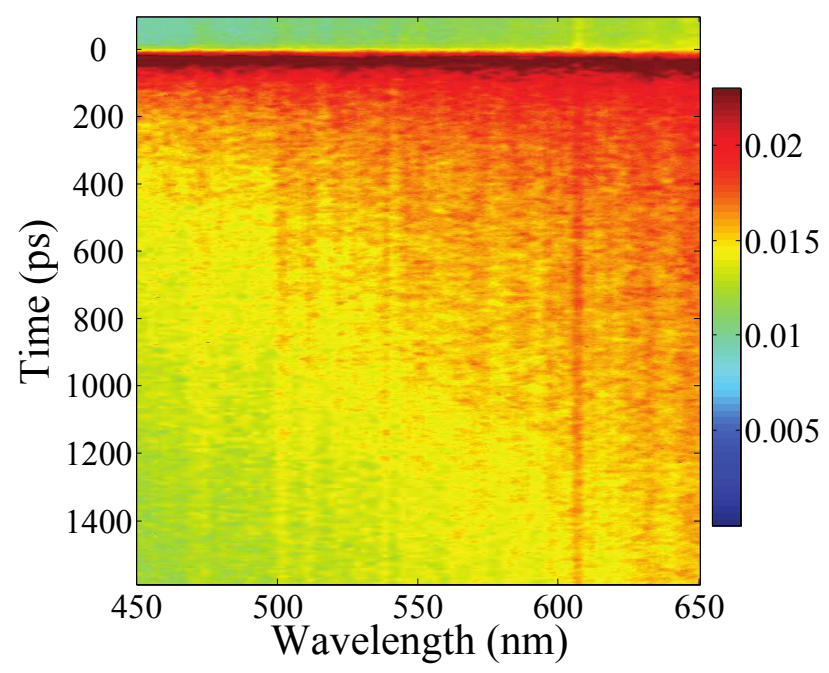


Figure S8. Two-axis plot of PL from a pellet sample with 343-nm photoexcitation at room temperature. The horizontal axis is the emission wavelength, and the vertical axis is the PL decay time. The excitation fluence is $13 \mu \mathrm{J} / \mathrm{cm}^{2}\left(2.2 \times 10^{13}\right.$ photons $/ \mathrm{cm}^{2} /$ pulse $)$.

Figure S9 shows the STE luminescence time profiles measured using TCSPC at various temperatures. The PL decays more slowly with decreasing temperature, which is consistent with turn-off of thermally assisted non-radiative channels.

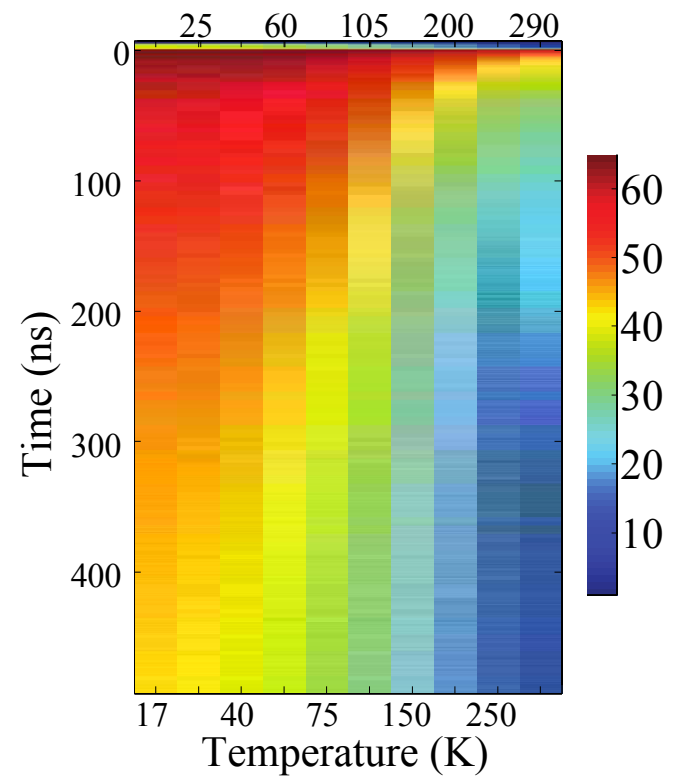

Figure S9. Two-dimensional STE luminescence time profiles measured at various temperatures. 


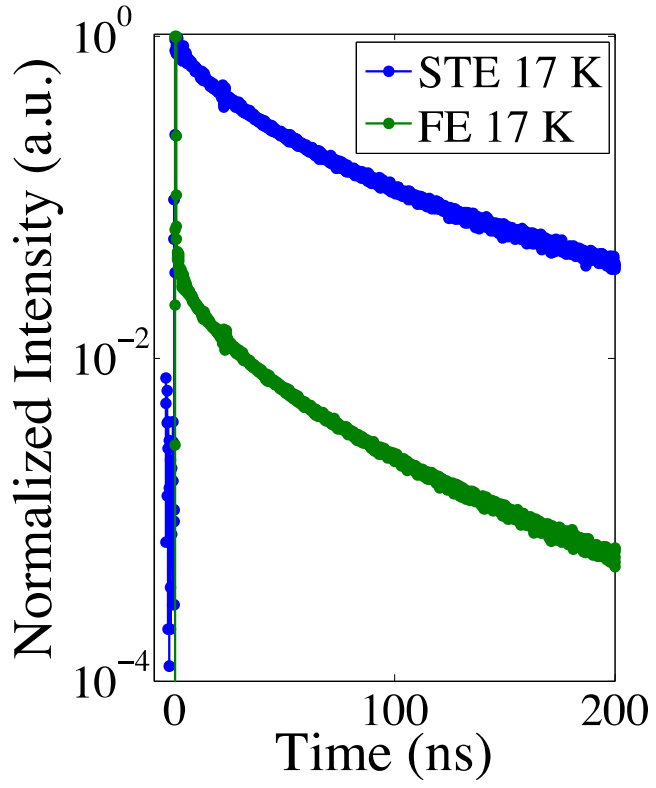

Figure S10. Time-dependent PL decay for STE and free exciton (FE) states at $17 \mathrm{~K}$.

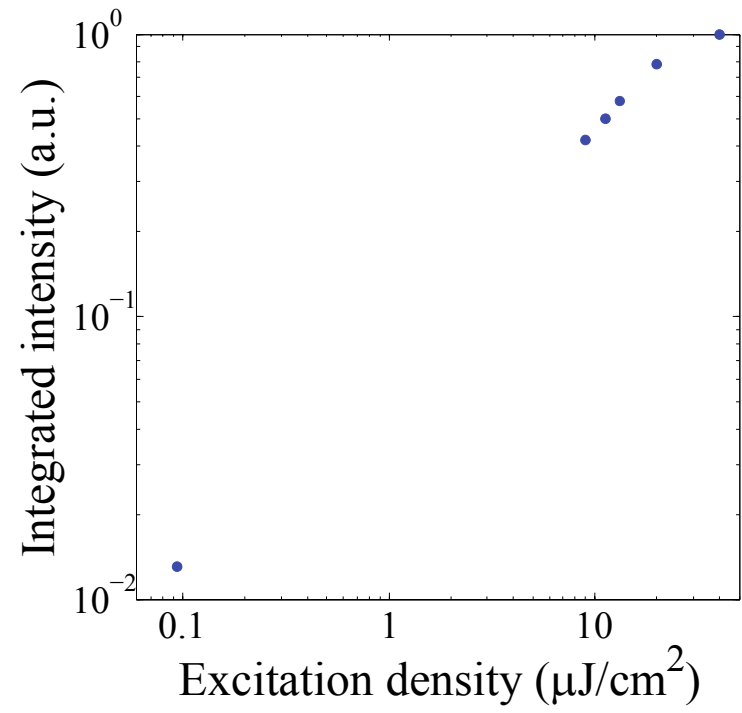

Figure S11. Fluence dependence of STE PL intensity (343-nm pump, 1.28 MHz). The last point (highest excitation density) deviates from linearity owing to material degradation. 


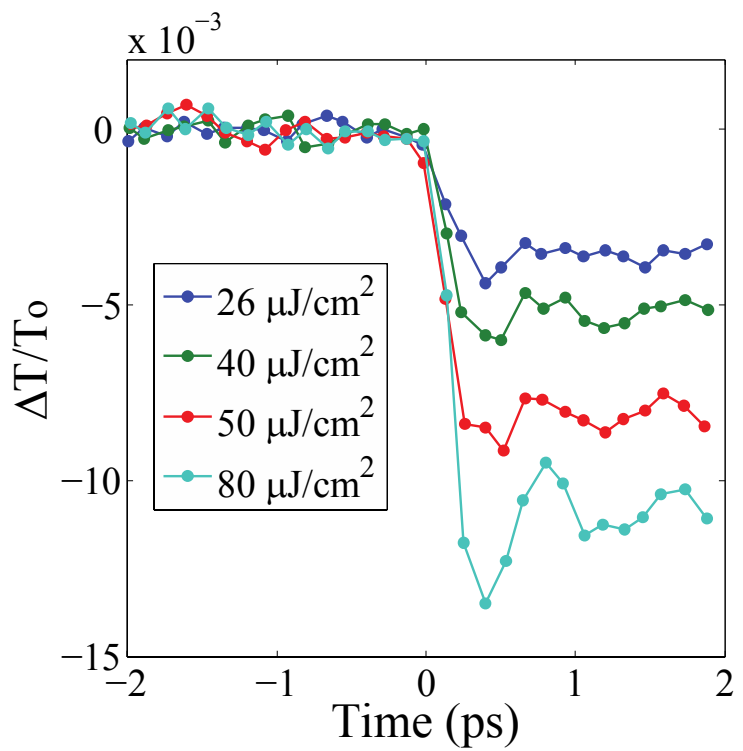

Figure S12. Fluence dependence of the TA signal (387-nm pump, 400-nm probe, $1 \mathrm{kHz}$ )

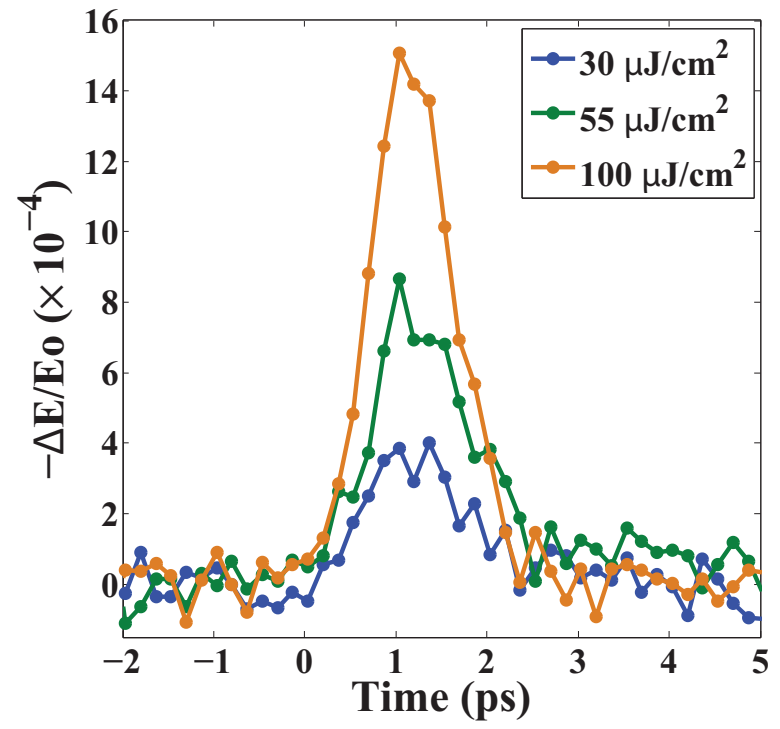

Figure S13. Relative change in the peak of $\mathrm{THz}$ transmission $\left(-\Delta E / E_{0}\right)$ due to transient photoconductivity at various pump fluences $(266-\mathrm{nm}, 1 \mathrm{kHz})$. 


\section{References}

(S1) Dohner, E. R.; Hoke, E. T.; Karunadasa, H. I. Self-Assembly of Broadband White-Light Emitters. J. Am. Chem. Soc. 2014, 136, 1718-1721.

(S2) Hu, T.; Wittenberg, J. S.; Lindenberg, A. M. Room Temperature Stabilization of Nanoscale Superionic Ag $\mathrm{Ag}_{2}$ Se. Nanotechnology 2014, 25, 415705-415709.

(S3) Takeda, J.; Nakajima, K.; Kurita, S.; Tomimoto, S.; Saito, S.; Suemoto, T. Time-resolved Luminescence Spectroscopy by the Optical Kerr-gate Method Applicable to Ultrafast Relaxation Processes. Phys. Rev. B 2000, 62, 10083-10085.

(S4) Mitzi, D. B. Thin-Film Deposition of Organic-Inorganic Hybrid Materials. Chem. Mater. 2001, 13, 3283-3298.

(S5) Wu, X.; Trinh, M. T.; Niesner, D.; Zhu, H.; Norman, Z.; Owen, J. S.; Yaffe, O.; Kudisch, B. J.; Zhu, X. Trap States in Lead Iodide Perovskites. J. Am. Chem. Soc. 2015, 137, 2089-2096. 